\title{
LOCATION OF THE ZEROS OF POLYNOMIALS WITH A PRESCRIBED NORM
}

\author{
BY
}

\section{Q. I. RAHMAN AND G. SCHMEISSER}

ABSTRACT. For monic polynomials $f_{n}(z)$ of degree $n$ with prescribed $L^{p}$ norm $(1 \leq p \leq \infty)$ on the unit circle or supremum nom on the unit interval we determine bounded regions in the complex plane containing at least $k$ ( 15 $k \leq n)$ zeros. We deduce our results from some new in equalities which are similar to an inequality of Vicente Gonçalves and relate the zeros of a polynomial to its norm.

The location of some or all the zeros of a polynomial

$$
f_{n}(z)=\sum_{j=0}^{n} a_{j} z^{j} \quad\left(a_{j} \in \mathbf{C}, 0 \leq j \leq n\right)
$$

in terms of its coefficients has been extensively studied (see [3, Chapters VIIIX]). We may as well investigate the location of the zeros of $f_{n}(z)$ in terms of a given norm. Such a problem is of interest in the theory of approximation [1, see $\$ 5]$. Since multiplication by a nonzero constant does not change the zeros of $f_{n}(z)$, norm alone cannot fumish any information regarding the location of any of the zeros. As a normalization we shall assume $f_{n}(z)=\sum_{j=0}^{n} a_{j} z^{j}$ to be monic, i.e. the coefficient of $z^{n}$ will be supposed to be 1 . As typical norms we consider $L^{p}$ norms on the unit circle and on the unit interval:

$$
\begin{aligned}
& \text { (1) }\left\|f_{n}\right\|_{p}=\left(\frac{1}{2 \pi} \int_{-\pi}^{\pi}\left|f_{n}\left(e^{i \theta}\right)\right|^{p} d \theta\right)^{1 / p} \quad(1 \leq p<\infty),\left\|f_{n}\right\|_{\infty}=\max _{-\pi \leq \theta<\pi}\left|f_{n}\left(e^{i \theta}\right)\right|, \\
& \text { (2) } \mathbb{M}_{p}\left(f_{n}\right)=\left(\frac{1}{2} \int_{-1}^{1}\left|f_{n}(x)\right|^{p} d x\right)^{1 / p} \quad(1 \leq p<\infty), \mathbb{M}_{\infty}\left(f_{n}\right)=\max _{-1 \leq x \leq 1}\left|f_{n}(x)\right|_{:}
\end{aligned}
$$

We wish to determine the radius $R(n, k, p, N)$ of the smallest disk centered at the origin containing at least $k(1 \leq k \leq n)$ zeros of every polynomial $f_{n}(z)=$

Received by the editors March $19,1973$.

AMS (MOS) subject classifications (1970). Primary 30A04, 30A06, 30A08; Secondary $30 \wedge 40,41 \wedge 10$.

Key words and phrases. Location of zeros, inequalities for polynomials, best approximation by polynomials, extremal problems. 
$z^{n}+\sum_{j=0}^{n-1} a_{j} z^{j}$ of degree $n$ with $\left\|f_{n}\right\|_{p}=N$. In case $N$ is given to be the supremum norm on the unit interval it tums out to be appropriate to find out the sum $\rho(n, k, \infty, N)$ of the semiaxes of the ellipse with foci at $-1,1$ and containing at least $k$ zeros of every polynomial $f_{n}(z)=z^{n}+\sum_{j=0}^{n-1} a_{j} z^{j}$ with $\mathbb{N}_{\propto}\left(f_{n}\right)=N$.

Quite a few results giving bounds for the zeros depending on the moduli of coefficients may be found in [3]. Since $\left\|f_{n}\right\|_{2}$ is expressible in terms of the coefficients some of these results may be used to determine estimates for $R(n, k, 2, N)$. For example, the polynomial $f_{n}(z)=z^{n}+\sum_{j=0}^{n-1} a_{j} z^{j}$ is known to have (see [3, (27, Formula 19)]) all its zeros in $|z|<\left(1+\sum_{j=1}^{n-1}\left|a_{j}\right|^{2}\right)^{1 / 2}$. Since $\left(1+\sum_{j=0}^{n-1}\left|a_{j}\right|^{2}\right)^{1 / 2} \equiv$ $\left\|f_{n}\right\|_{2}$ this shows that $R(n, n, 2, N)<N$. But $R(n, n, 2, N)$ is easily seen to be equal to the positive root $R(n, N)$ of the equation

$$
R^{2 n}-\left(N^{2}-1\right) \sum_{\nu=0}^{n-1} R^{2 \nu}=0 .
$$

In fact, if $\zeta$ is a zero of the polynomial $f_{n}(z)=z^{n}+\sum_{j=0}^{n-1} a_{j} z^{j}$ with $\left\|f_{n}\right\|_{2}=N$, then

$$
|\zeta|^{n}=\left|-\sum_{j=0}^{n-1} a_{j} \zeta\right| \leq\left\{\left(N^{2}-1\right) \sum_{j=0}^{n-1}|\zeta|^{2 j}\right\}^{1 / 2}
$$

which shows that the largest positive root $R(n, N)$ of (3) is a bound for the moduli of all the zeros. Given $N \geq 1$

$$
f_{n}(z)=z^{n}-\left(N^{2}-1\right) \sum_{j=0}^{n-1} \frac{z^{j}}{\{R(n, N)\}^{n-j}}
$$

is a polynomial of degree $n$ with $\left\|f_{n}\right\|_{2}=N$ and having a zero on $|z|=R(n, N)$. Substituting $R^{2}=N^{2}-\alpha$ in (3) we get $\left(N^{2}-1\right) / N^{2 n}=\alpha\left(1-a / N^{2}\right)^{n}$. Hence for fixed $n$ and large $N, \alpha=O\left(1 / N^{2(n-1)}\right)$, i.e.

$$
R=N\left(1-O\left(N^{-2 n}\right)\right)
$$

If $1 \leq k<n$ an upper estimate for $R(n, k, 2, N)$ can be deduced from the following result of Vicente Gonçalves ([10], also see [4] and [3, Exercise 4, p. 130]).

Theorem A. Consider tbe polynomial $f_{n}(z)=z^{n}+\sum_{j=0}^{n-1} a_{j} z^{j}$ and let $\zeta_{1}{ }^{\prime} \zeta_{2}$, $\ldots, \zeta_{n}$ denote the zeros of $f_{n}(z)$ in an arbitrary order. Then for $1 \leq k \leq n$

$$
\left|\zeta_{1} \zeta_{2} \cdots \zeta_{k-1}\right|^{2}+\left|\zeta_{k} \zeta_{k+1} \cdots \zeta_{n}\right|^{2} \leq\left\|f_{n}\right\|_{2}^{2}
$$

where for $k=1$ the first term on the left-hand side is to be replaced by 1 . 
In particular

$$
R(n, 1,2, N) \leq\left(N^{2}-1\right)^{1 /(2 n)}
$$

The example $f_{n}(z)=z^{n}+\left(N^{2}-1\right)^{1 / 2}(N \geq 1)$ shows that, in fact,

$$
R(n, 1,2, N) \equiv\left(N^{2}-1\right)^{1 /(2 n)}
$$

For $p \neq 2$ the known bounds for the moduli of the zeros in terms of the coefficients do not seem to be of much avail. But Jensen's formula gives (see [9, $\$ 3.61]$, and $[7, \$ 9$, p. 21])

$$
R(n, k, p, N) \leq N^{1 /(n-k+1)} \quad(1 \leq k \leq n, 1 \leq p \leq \infty)
$$

which for $p=2$ is weaker than what is obtainable from (5). So, in order to improve on (7) we seek to extend (5) to values of $p$ other than 2 . In the case $p=\infty$ this is done with the help of the following inequality due to Visser [11]:

$$
\left|a_{0}\right|+\left|a_{n}\right| \leq \max _{|z|=1}\left|\sum_{j=0}^{n} a_{j} z^{j}\right| .
$$

For $1<p \leq 2$ we use

$$
\left(\left|a_{0}\right|^{q}+\left|a_{n}\right|^{q}\right)^{1 / q} \leq\left(\frac{1}{2 \pi} \int_{-\pi}^{\pi}\left|\sum_{j=0}^{n} a_{j} e^{i j \theta}\right|^{p} d \theta\right)^{1 / p}
$$

$$
\left(1<p \leq 2, p^{-1}+q^{-1}=1\right)
$$

which is a weaker form of the Hausdorff-Young inequality [13, p. 101].

From (8), (9) we deduce the following generalization of the inequality of Vicente Gonçalves (loc. cit.).

Theorem 1. Consider the polynomial $f_{n}(z)=z^{n}+\sum_{j=0}^{n-1} a_{j} z^{j}$ and let $\zeta_{1}, \zeta_{2}$, $\cdots, \zeta_{n}$ denote the zeros of $f_{n}(z)$ in an arbitrary order. Then for $1 \leq k \leq n$

$$
\left(\left|\zeta_{1} \zeta_{2} \cdots \zeta_{k-1}\right|^{q}+\left|\zeta_{k} \zeta_{k+1} \cdots \zeta_{n}\right|^{q}\right)^{1 / q} \leq\left\|f_{n}\right\|_{p}
$$

$$
\left(p=\infty \text { or } 1<p \leq 2, p^{-1}+q^{-1}=1\right)
$$

where for $k=1$ the term $\left|\zeta_{1} \zeta_{2} \cdots \zeta_{k-1}\right|^{q}$ on the left-hand side is to be replaced by 1 .

Proof. It is clear that if Theorem 1 holds for monic polynomials not vanishing at the origin then it also holds for those which have a simple or a multiple zero at the origin. So let $\zeta_{k}, \zeta_{k+1}, \cdots, \zeta_{n}$ be different from zero and apply (8), (9) to 


$$
g_{n}(z)=f_{n}(z) \prod_{j=k}^{n}\left(\frac{\bar{\zeta}_{j} z-1}{z-\zeta_{j}}\right) \text {. }
$$

Since $\left|f_{n}(z)\right|=\left|g_{n}(z)\right|$ for $|z|=1$ we get

$$
\begin{aligned}
\left(\left|\frac{a_{0}}{\zeta_{k} \zeta_{k+1} \cdots \zeta_{n}}\right|^{q}+\left|\bar{\zeta}_{k} \bar{\zeta}_{k+1} \cdots \bar{\zeta}_{n}\right|^{q}\right)^{1 / q} \leq & \left\|f_{n}\right\|_{p} \\
& \left(p=\infty, 1<p \leq 2, p^{-1}+q^{-1}=1\right)
\end{aligned}
$$

which is equivalent to (10).

Remark 1: The example $f_{n}(z)=z^{n}+1$ shows that $(10)$ is false for $2<p<\infty$. In fact, for $2<p<\infty$

$$
\left\|1 / 2\left(z^{n}+1\right)\right\|_{p}^{p}<\left\|1 / 2\left(z^{n}+1\right)\right\|_{2}^{2}=1 / 2
$$

so that $\left\|z^{n}+1\right\|_{p}<2(1 / 2)^{1 / p}=2^{1 / q}=\left(1^{q}+1^{q}\right)^{1 / q}$.

Remark 2 (The case of equality in (10)). In (8) equality holds if and only if $a_{j}=0$ for $j \neq 0, n$. The same is true of (9) if $p=2$. If $1<p<2$ and $a_{n} \neq 0$ then by a result in [13] (see (2.25) on p. 105) there is strict inequality in (9) unless $a_{j}=0, j=0,1, \cdots, n-1$. Taking these facts into account and excluding the trivial case of $f_{n}(z)=z^{n}$ the proof of Theorem 1 shows that in (10) equality is not possible for $1<p<2$ and that for $p=\alpha, p=2$ equality holds if and only if $f_{n}(z)$ has the form

$$
\prod_{\nu=1}^{k-1}\left(z-R^{-1} e^{i\left(a+\phi_{\nu}\right)}\right) \prod_{\nu=k}^{n}\left(z-R e^{i\left(a_{+} \phi_{\nu}\right)}\right)
$$

where $\left\{e^{i \phi_{\nu} n}{ }_{\nu=1}^{n}\right.$ are the $n$th roots of unity in arbitrary order, $R$ is an arbitrary positive number and $\alpha$ an arbitrary real number.

Remark 3. It is seen from Jensen's formula that inequality (10) may be extended to cover the case $p=1$ by replacing the left-hand side by its limiting value (as $p \rightarrow 1) \max \left(\left|\zeta_{1} \zeta_{2} \cdots \zeta_{k-1}\right|,\left|\zeta_{k} \zeta_{k+1} \cdots \zeta_{n}\right|\right)$.

Remark 4. If $f_{n}(z)$ is a polynomial of degree $n$ then by an inequality of Zygmund [12] we have

$$
\int_{0}^{2 \pi}\left|f_{n}^{\prime}\left(e^{i \theta}\right)\right|^{p} d \theta \leq \gamma_{p} n^{p} \int_{0}^{2 \pi}\left|\operatorname{Re} f_{n}\left(e^{i \theta}\right)\right|^{p} d \theta \quad(p \geq 1)
$$

where

$$
\gamma_{p}=\sqrt{\pi} \Gamma(1 / 2 p+1) / \Gamma(1 / 2(p+1))
$$

Applying Theorem 1 to $f_{n}^{\prime}(z)$ and noting that $\lim _{p \rightarrow \infty} \gamma_{p}^{1 / p}=1$ we obtain the following result on the location of critical points of $f_{n}(z)$. 
Corollary 1. Denote by $\eta_{1}, \eta_{2}, \cdots, \eta_{n-1}$ the critical points of a monic polynomial $f_{n}(z)$ of degree $n$. Then for $1 \leq k \leq n-1$

$$
\begin{aligned}
\left(\left|\eta_{1} \eta_{2} \cdots \eta_{k-1}\right|^{q}+\left|\eta_{k} \eta_{k+1} \cdots \eta_{n-1}\right|^{q}\right)^{1 / q} \leq \gamma_{p}^{1 / p}\left\|\operatorname{Re} f_{n}\right\|_{p} & \\
& \left(1 \leq p \leq 2, p^{-1}+q^{-1}=1\right)
\end{aligned}
$$

and

$$
\left|\eta_{1} \eta_{2} \cdots \eta_{k-1}\right|+\left|\eta_{k} \eta_{k+1} \cdots \eta_{n-1}\right| \leq\left\|\operatorname{Re} f_{n}\right\|_{\infty}
$$

For $k=1$ the first term on the left band side in both inequalities is to be replaced by 1.

The next result is an immediate consequence of.Theorem 1.

Corollary 2. If $\zeta_{1}, \zeta_{2}, \cdots, \zeta_{n}$ are the zeros of $f_{n}(z)=z^{n}+\sum_{j=0}^{n-1} a_{j} z^{j}$ arranged in increasing order of moduli then we have

$$
\left|\zeta_{1}\right| \leq R(n, 1, p, N) \leq\left(N^{q}-1\right)^{1 /(q n)}
$$

$$
\left(p=\infty \text { or } 1<p \leq 2, p^{-1}+q^{-1}=1\right)
$$

and for $2 \leq k \leq n$

$$
\left|\zeta_{k}\right| \leq\left|\zeta_{k} \zeta_{k+1} \cdots \zeta_{n}\right|^{1 /(n-k+1)}
$$

$$
\begin{aligned}
& \leq\left(1 / 2\left\|f_{n}\right\|_{p}^{q}+1 / 2\left(\left\|f_{n}\right\|_{p}^{2 q}-4\left|a_{0}\right|^{2}\right)^{1 / 2}\right)^{1 /\{q(n-k+1)\}} \\
& \quad\left(p=\infty \text { or } 1<p \leq 2, p^{-1}+q^{-1}=1\right) .
\end{aligned}
$$

Inequalities (12), (13) are best possible for $p=\infty, 2$. In particular

$$
R(n, 1, \infty, N) \equiv(N-1)^{1 / n} \quad(N \geq 1)
$$

wbicb is attained for $f_{n}(z)=z^{n}+(N-1)$.

Unfortunately the bound in (13) depends on $\left|a_{0}\right|$. But, in any case, it is at least as good as (7). It may be noted that for large $N$ and $p=2, \infty$ there is not much room for improvement in (7). To see this let $f_{n-1}(z)=z^{n-1}+\sum_{j=0}^{n-2} a_{j} z^{j}$ be a monic polynomial of degree $n-1$ with $\left\|f_{n-1}\right\|_{p}=N$. Then $g(z)=z f_{n-1}(z)$ has at least $k$ zeros in $|z| \leq R(n-1, k-1, p, N)$. Since $g(z)$ is a monic polynomial of degree $n$ with $\|g\|_{p}=N$ we have

$$
R(n, k, p, N) \geq R(n-1, k-1, p, N) \text {. }
$$

This leads us to the conclusion that $R(n, k, p, N) \geq R(n-k+1,1, p, N)$, and by $\left(6^{*}\right),\left(12^{*}\right)$ respectively, we get 


$$
R(n, k, 2, N) \geq\left(N^{2}-1\right)^{1 /\{2(n-k+1)\}}, \quad R(n, k, \infty, N) \geq(N-1)^{1 /(n-k+1)}
$$

showing that the bounds for $R(n, k, 2, N), R(n, k, \infty, N)$ obtainable from (13) are not too bad for large $N$.

With the help of Theorem 1 we obtain a slight improvement of (7) (it is only for sake of simplicity that we restrict ourselves to the case of supremum norm).

Let $\zeta_{1}$ be a zero of smallest modulus of $f_{n}(z)=z^{n}+\sum_{j=0}^{n-1} a_{j} z^{j}$. Then by (10) $f_{n}(z)$ has at least two zeros in

$$
|z| \leq\left(\left\|f_{n}\right\|_{\infty}-\left|\zeta_{1}\right|\right)^{1 /(n-1)} \text {. }
$$

On the other hand, if $f_{n-1}(z)=f_{n}(z) /\left(z-\zeta_{1}\right)$ then by $(12), f_{n-1}(z)$ has at least one and $f_{n}(z)$ at least two zeros in

$$
|z| \leq\left(\frac{\left\|f_{n}\right\|_{\infty}}{\sqrt{1-\mid \zeta_{1} \|^{-1}}-1}\right)^{1 /(n-1)} \quad\left(\left|\zeta_{1}\right| \neq 1\right) .
$$

Hence, whatever $\left|\zeta_{1}\right|$ may be, $f_{n}(z)$ has at least two zeros in

$$
|z| \leq\left\{1 / 2\left(\left\|f_{n}\right\|_{\infty}-2\right)+1 / 2 \sqrt{\left(\left\|f_{n}\right\|_{\infty}+2\right)^{2}-4}\right\}^{1 /(n-1)},
$$

i.e. we have

Corollary 3. $R(n, 2, \infty, N) \leq\left\{1 / 2(N-2)+1 / 2 \sqrt{(N+2)^{2}-4}\right\}^{1 /(n-1)}$.

Thus, $(R(n, 2, \infty, N))^{n-1}$ has an upper bound independent of $n$ which we denote by $r_{2}(N)$-the subscript 2 refers to 2 zeros. Now suppose that an upper bound $r_{k}(N)$ (independent of $\left.n\right)$ for $\left(R(n, k, \infty, N)^{n-k+1}\right.$ has been found. Then $f_{n}(z) /\left(z-\zeta_{1}\right)$ has at least $k$ and $f_{n}(z)$ at least $k+1$ zeros in

$$
D_{1}\left(\zeta_{1}\right)=\left\{z:|z| \leq\left(r_{k}\left(N /|1-| \zeta_{1}||\right)\right)^{1 /(n-k)}\right\}, \quad\left(\left|\zeta_{1}\right| \neq 1\right) .
$$

On the other hand we may conclude from $(10)$ that $f_{n}(z)$ has at least $k+1$ zeros in

$$
D_{2}\left(\zeta_{1}\right)=\left\{z:|z| \leq\left(N-\left|\zeta_{1}\right|^{k}\right)^{1 /(n-k)}\right\} .
$$

Comparing the radii of $D_{1}\left(\zeta_{1}\right)$ and $D_{2}\left(\zeta_{1}\right)$ we see that $r_{k+1}(N)$ may be taken to be equal to $N-\left(\lambda_{k}(N)\right)^{k}$ where $\lambda_{k}(N)$ is the smallest positive root of the equation $r_{k}(N /(1-\lambda))=N-\lambda^{k}$. Thus

$$
R(n, k+1, \infty, N) \leq\left(N-\left(\lambda_{k}(N)\right)^{k}\right)^{1 /(n-k)}
$$

which is an improvement on (7).

As pointed out in Remark 1 inequality (10) does not hold for $2<p<\infty$. 
Since $\left\|f_{n}\right\|_{p}$ is a nondecreasing function of $p$ we obtain from Theorem A

$$
\left(\left|\zeta_{1} \zeta_{2} \cdots \zeta_{k-1}\right|^{2}+\left|\zeta_{k} \zeta_{k+1} \cdots \zeta_{n}\right|^{2}\right)^{1 / 2} \leq\left\|f_{n}\right\|_{p} \quad(2 \leq p<\infty)
$$

and in particular

$$
R(n, 1, p, N) \leq\left(N^{2}-1\right)^{1 / 2 n} \quad(2 \leq p<\infty) .
$$

Another result like inequality (10) but valid for $1 \leq p<\propto$ is the following.

Theorem 2. In the notations of Theorem 1 we have for $1 \leq p<\propto$ and $1 \leq k$ $\leq n$

$$
\left|\zeta_{1} \zeta_{2} \cdots \zeta_{k-1}\right|+\left|\zeta_{k} \zeta_{k+1} \cdots \zeta_{n}\right| \leq \gamma_{p}^{1 / p}\left\|f_{n}\right\|_{p}
$$

where $\gamma_{p}$ is given by (11). For $k=1$ the first term on the left-hand side of (15) is to be replaced by 1 .

This result can be deduced from the following lemma (see [5, Theorem 2]) in the same way as Theorem 1 was deduced from (8), (9).

Lemma 1. If $f(z)=\sum_{j=0}^{n} a_{j} z^{j}$ is a polynomial of degree $n$ and $a_{u^{\prime}} a_{v}(u<v)$ are two coefficients such that for no other coefficients $a_{w} \neq 0$ do we bave $w \equiv u \bmod (v-u)$, then for every $p \geq 1,\left|a_{u}\right|+\left|a_{v}\right| \leq \gamma_{p}^{1 / p}\|f\|_{p}$ where $\gamma_{p}$ is given by (11).

From (15) it follows that

$$
R(n, 1, p, N) \leq\left(\gamma_{p}^{1 / p} N-1\right)^{1 / n} \quad(1 \leq p<\infty)
$$

The limiting case as $p \rightarrow \infty$ of (16) agrees with $\left(12^{*}\right)$. The bound in (16) is attained for $f_{n}(z)=z^{n}+e^{i \alpha}$, a real.

Comparing (16) with (12) for $1<p<2$ and with (14) for $2<p<\infty$ it is seen, that in both cases the bound for $R(n, 1, p, N)$ given by (16) is better or worse than the other one depending on the value of $N$.

We now turn to the study of the location of zeros of a monic polynomial $f_{n}(z)$ in terms of $\mathbb{N}_{p}\left(f_{n}\right)$. As $\mathbb{R}_{2}\left(f_{n}\right)$ may be expressed in terms of the moduli of the coefficients in the Legendre-development of $f_{n}(z)$ regions containing at least $k(1 \leq k \leq n)$ zeros of $f_{n}(z)$ may be obtained from the following (specialized versions of) known results.

Theorem B [8]. For $f_{n}(z)=\Pi_{\nu=1}^{n}\left(z-\zeta_{\nu}\right)$ we have

$$
\sum_{\nu=1}^{n}\left(\frac{d_{n}\left(\zeta_{n}\right) d_{n}\left(\zeta_{n-1}\right) \cdots d_{n}\left(\zeta_{\nu}\right)}{\lambda_{\nu-1}}\right) 2 \leq\left(\prod_{2}\left(f_{n}\right)\right)^{2}-\lambda_{n}^{-2}
$$

where 


$$
\lambda_{0}=1, \quad \lambda_{\nu}=\frac{1}{2^{\nu}}\left(\begin{array}{l}
2 \nu \\
\nu
\end{array}\right) \sqrt{2 \nu+1} \quad(1 \leq \nu \leq n)
$$

and $d_{n}(z)$ denotes the distance of $z$ from the span of the zeros of the nth Legendre polynomial.

Theorem C [2]. In the notations of Theorem B we have

$$
\frac{1}{\lambda_{n-1}} \sum_{\nu=1}^{n} d_{n}\left(\zeta_{\nu}\right) \leq\left(\left(\mathbb{P}_{2}\left(f_{n}\right)\right)^{2}-\lambda_{n}^{-2}\right)^{1 / 2}
$$

For the purpose of determining the location of $k(1 \leq k \leq n)$ zeros of $f_{n}(z)=$ $z^{n}+\sum_{\nu=0}^{n-1} a_{\nu} z^{\nu}$ in terms of $\mathbb{M}_{\propto}\left(f_{n}\right)$ we prove the following inequality which is somewhat similar to (10).

Theorem 3. Let $f_{n}(z)=\Pi_{\nu=1}^{n}\left(z-\zeta_{\nu}\right)$ be a real polynomial of degree $n$ wbich does not change sign on the unit interval. If $R_{\nu}$ denotes the sum of the semiaxes of the ellipse $\mathcal{E}\left(R_{\nu}\right)$ with foci at $+1,-1$ and passing through the point $\zeta_{\nu}$ $(\nu=1,2, \cdots, n)$ then for $1 \leq k \leq n$

$$
\frac{R_{1} R_{2} \cdots R_{k-1}}{R_{k} R_{k+1} \cdots R_{n}}+\frac{R_{k} R_{k+1} \cdots R_{n}}{R_{1} R_{2} \cdots R_{k-1}} \leq 2\left(2^{n-1} \mathbb{M}_{\alpha}\left(f_{n}\right)-1\right) .
$$

For $k=1$ the product $R_{1} R_{2} \cdots R_{k-1}$ is to be replaced by 1 .

Proof. Under the hypothesis $\left|f_{n}(\cos \theta)\right|$ is a nonnegative trigonometric polynomial of degree $n$. By a well-known theorem of Fejér and Riesz (see [6, p. 117]) there exists a polynomial $F_{n}(z)=A_{n} \Pi_{\nu=1}^{n}\left(z-Z_{\nu}\right)$ with $\left|Z_{\nu}\right| \geq 1$ and $Z_{\nu}^{-1}+Z_{\nu}=2 \zeta_{\nu}(\nu=1,2, \cdots, n)$ such that

$$
\left|f_{n}(\cos \theta)\right|=\left|F_{n}\left(e^{i \theta}\right)\right|^{2} \quad(\theta \text { real }) .
$$

Replacing $\cos \theta$ by $1 / 2\left(e^{i \theta}+e^{-i \theta}\right)$ and equating the coefficients of $e^{i n \theta}$ on the two sides of (18) we get $1 / 2^{n}=\left|A_{n}\right|^{2} \Pi_{\nu=1}^{n}\left|Z_{\nu}\right|$. Hence, by Theorem 1 we have

$$
\left|Z_{1} Z_{2} \cdots Z_{k-1}\right|+\left|Z_{k} Z_{k+1} \cdots Z_{n}\right| \leq\left(2^{n} \prod_{\nu=1}^{n}\left|Z_{\nu}\right|\right)^{1 / 2}\left\|F_{n}\right\|_{\infty}
$$

which is equivalent to

$$
\left|\frac{Z_{1} Z_{2} \cdots Z_{k-1}}{Z_{k} Z_{k+1} \cdots Z_{n}}\right|+\left|\frac{Z_{k} Z_{k+1} \cdots Z_{n}}{Z_{1} Z_{2} \cdots Z_{k-1}}\right| \leq 2\left(2^{n-1} \Re_{\infty}\left(f_{n}\right)-1\right) .
$$

Inequality (17) follows from this on noting that $\zeta_{\nu}$ lies on the ellipse $\mathcal{E}\left(\left|Z_{\nu}\right|\right)$ $(\nu=1,2, \cdots, n)$.

Remark 5 (The case of equality in (17)). Taking into account the case of 
equality in (10) (as discussed in Remark 2) and the identity (18) we easily see that for $k=1$ and given $N=\mathbb{M}_{\propto}\left(f_{n}\right) \geq 1 / 2^{n-2}$ equality holds in (17) for

$$
f_{n}(z)=\frac{1}{2^{n-1}}\left(T_{n}(z) \pm\left(2^{n-1} N-1\right)\right)
$$

where $T_{n}(x)=\cos n$ arc $\cos x$ is the $n$th Chebyshev polynomial. If $2 \leq k \leq n$ equality holds only for $f_{n}(z)=2^{-(n-1)}\left(T_{n}(z) \pm 1\right)$.

We may apply Theorem 3 to the polynomial $f_{n}(z) \overline{f_{n}(\bar{z})}$ of degree $2 n$ to obtain the following

Corollary 4. If $f(z)=\Pi_{\nu=1}^{n}\left(z-\zeta_{\nu}\right)$ is a polynomial of degree $n$ then with $R_{\nu}(\nu=1,2, \cdots, n)$ as defined in Theorem 3 we have for $1 \leq k \leq n$

$$
\frac{R_{1} R_{2} \cdots R_{k-1}}{R_{k} R_{k+1} \cdots R_{n}}+\frac{R_{k} R_{k+1} \cdots R_{n}}{R_{1} R_{2} \cdots R_{k-1}} \leq 2^{n} \mathbb{M}_{\propto}\left(f_{n}\right) \text {. }
$$

For $k=1$ the product $R_{1} R_{2} \cdots R_{k-1}$ is to be replaced by 1. Equality bolds for $f_{n}(z)=2^{-(n-1)} T_{n}(z)$ where $T_{n}(z)$ is the nth Chebyshev polynomial.

From Theorem 3 (in conjunction with Remark 5) and Corollary 4 we may deduce the following results.

Corollary 5. If $\rho^{*}(n, k, \infty, N)$ denotes the sum of the semiaxes of the ellipse with foci at $+1,-1$ and containing at least $k$ zeros of every real monic polynomial $f_{n}(z)$ with $\mathbb{M}_{\propto}\left(f_{n}\right)=N$ then

(20) $\rho^{*}(n, 1, \propto, N) \equiv \begin{cases}1 & \text { for } 2^{-(n-1)} \leq N \leq 2^{-(n-2)}, \\ \left(\sqrt{2^{n-2} N}+\sqrt{2^{n-2} N-1}\right)^{2 / n} & \text { for } N \geq 2^{-(n-2)},\end{cases}$

where the polynomials $f_{n}(z)$ given in (19) are extremal.

Corollary 6. Let $f_{n}(z)=\prod_{\nu=1}^{n}\left(z-\zeta_{\nu}\right)$ be a real polynomial which does not change sign in $(-1,1)$. If $\mathbb{M}_{\alpha}\left(f_{n}\right)=N$ then $f_{n}(z)$ has at least $k(1 \leq k \leq n)$ zeros in

$$
\mathcal{E}\left(\left(\sqrt{2^{n-2} N}+\sqrt{2^{n-2} N-1}\right)^{2 /(n-k+1)}\right) \quad\left(N \geq 2^{-(n-2)}\right) .
$$

Proof. Let the zeros of $f_{n}(z)$ be arranged in such a way that the corresponding numbers $R_{\nu}$ are nondecreasing in magnitude and put

$$
S=R_{n} R_{n-1} \cdots R_{k} \cdot \frac{R_{k-1}}{R_{k-2}} \cdot \frac{R_{k-3}}{R_{k-4}} \cdots \omega,
$$

where $\omega$ is equal to 1 for $k=1$ and equal to $R_{2} / R_{1}$ or $R_{1}$ according as $k \neq 1$ is odd or even respectively. Since (17) holds for every arrangement of the numbers $R_{\nu}$ we get $S+S^{-1} \leq 2\left(2^{n-1} N-1\right)$. From this Corollary 6 follows on noting 
that $R_{k}^{n-k+1} \leq R_{n} R_{n-1} \cdots R_{k} \leq S$. We observe that the monic polynomials $f_{n}(z)$ having no sign change in $(-1,1)$ and deviating least from zero on the unit interval are $2^{-(n-1)}\left(T_{n}(z) \pm 1\right)$ with deviation $N=2^{-(n-2)}$.

In the same way we can deduce from Corollary 4 the following result.

Corollary 7. If $\rho(n, k, \infty, N)$ is as defined in the beginning of this paper we bave

(21) $\rho(n, k, \infty, N) \leq\left(2^{n-1} N+\sqrt{\left(2^{n-1} N\right)^{2}-1}\right)^{1 /(n-k+1)} \quad\left(N \geq 2^{-(n-1)}\right)$.

The Corollaries 5-7 add to the information available to us from the work of S. N. Bernštein $[1, \S 5]$.

\section{REFERENCES}

1. S. N. Bemšteìn and C. de la Vallée Poussin, L'approximation, Chelsea, New York, 1970. MR $40 \# 2511$.

2. A. Giroux, Estimates for the imaginary parts of the zeros of a polynomial, Proc. Amer. Math. Soc. 44 (1974), 61-67.

3. M. Marden, Geometry of polynomials, 2nd ed., Mathematical Surveys, no. 3, Amer. Math. Soc., Providence, R. I., 1966. MR 37 \#1562.

4. A. M. Ostrowski, On an inequality of J. Vicente Gonçalves, Univ. Lisboa Revista Fac. Ci. A (2) 8 (1960), 115-119. MR 26 \#2585.

5. Q. I. Rahman, Inequalities conceming polynomials and trigonometric polynomials, J. Math. Anal. Appl. 6 (1963), 303-324. MR 27 \#2771.

6. F. Riesz and B. Sz.-Nagy, Leqons d'analyse fonctionnelle, 2nd ed., Akad. Kiadó, Budapest, 1953; English transl.,. Functional analysis, Ungar, New York, 1955. MR 15, 132; $17,175$.

7. W. Specht, Algebraische Gleichungen mit reellen oder komplexen Koeffizienten, Enzyklopädie der math. Wissenschaften: Mit Einschluss ihrer Anwendungen, Band I, 1, Heft 3, Teil II, Teubner Verlagsgesellschaft, Stuttgart, 1958. MR 21 \#5008.

8. - unpublished manuscript.

9. E. C. Titchmarsh, The theory of functions, 2nd ed., Oxford Univ. Press, Oxford, 1939.

10. J. Vicente Gonçalves, L'inégalité de W. Specht, Univ. Lisboa Revista Fac. Ci. A (2) 1 (1950), 167-171. MR 12, 605.

11. C. Visser, A simple proof of certain inequalities concerning polynomials, Nederl. Akad. Wetensch. Proc. 48, 276-281 = Indag. Math. 7 (1945), 81-86. MR 7, 440.

12. A. Zy gmund, Two notes on inequalities, J. Math. Phys. Mass. Inst. Tech. 21 (1942), 117-123. MR 4, 135.

13. —-, Trigonometric series. Vol. II, Cambridge Univ. Press, New York, 1959. MR $21 \# 6498$.

DEPARTMENT OF MATHEMATICS, UNIVERSITY OF MONTREAL, MONTREAL, QUEBEC, CANADA (Current address of Q. I. Rahman)

Current address (G. Schmeisser): Mathematisches Institut der Universität, ErlangenNürnberg, 852 Erlangen, Bismarckstrasse $1 \frac{1}{2}$, West Germany 